\author{
Jan Koornneef \\ Space Telescope Science Institute \\ Homewood Campus, \\ Baltimore, MD 21218
}

Abstract: From radio observations it has been known for some time that a relatively large fraction of the total mass of the Magellanic clouds is in the form of atomic Hydrogen. In contrast, optical photometry shows that both galaxies suffer rather little obscuration by dust, except in regions where the stellar population indicates recent star formation. However, the possibilities of discussing gas-to-dust ratios have been limited by the poor spatial resolution of the $21 \mathrm{~cm} \mathrm{HI}-$ observations and uncertainties with respect to the relative location of the various components (gas, dust and stars).

Over the last few years, observations of about thirty cloud supergiants with the International Ultraviolet Explorer have yielded estimates of the neutral Hydrogen column densities from measurements of the Lyman $\alpha$ absorption profile in the ultraviolet spectra which were also used to study the wavelength dependence of the extinction due to the intervening dust. The extinction laws have been shown to be strongly anomalous in both of the MCs. It now appears certain that the gas-to-dust ratios in the MCs are significantly larger than those in our Galaxy. If expressed by the parameter $\mathrm{N}_{\mathrm{H}} / \mathrm{E}_{\mathrm{B}-\mathrm{V}}$, the LMC ratio is approximately four times larger than the Galactic value, whereas the SMC value is about seventeen times the Galactic ratio.

The presently available data fit into the following scheme: Galaxy $\rightarrow$ LMC $\longrightarrow$ SMC: decreasing heavy-element abundances, decreasing strength of the $2200 \AA$ dust-feature, increasing gas-to-dust ratio and increasing far-ultraviolet extinction.

\title{
RADIO OBSERVATIONS
}

Neutral hydrogen observations of the Magellanic Clouds have been available for quite some time. In 1966, McGee and Milton published the $21 \mathrm{~cm}$ Parkes Survey of the IMC which has a spatial resolution of $1^{\prime}$. A similar HI map for the SMC was published by Hindman (1967). The SMC data were re-examined by McGee and Newton (1981) and the same authors 
added new SMC-HI observations in 1982. Total hydrogen masses from these studies are roughly $510^{8} \mathrm{M}_{\odot}$ for each of the galaxies, which translates into a typical column density of $\mathrm{N}_{\mathrm{HI}}=4.510^{21} \mathrm{~cm}^{-2}$ for the SMC (Azzopardi and Vigneau 1977) and somewhat smaller values for the LMC because of its larger size. The local gas profile near zero velocity gives a galactic foreground column density in the range of $\mathrm{N}_{\mathrm{HI}}=3.2 \pm 1.310^{20} \mathrm{~cm}^{-2}$ in the direction of the IMC (McGee, Newton and Morton, 1983). Towards the SMC, local hydrogen is mostly between 2.7 and $3.510^{20}$ (MCGee, priv. comm.). In both MCs, the HI profiles contain a wealth of velocity information indicative of the existence of very large scale structures. In the context of the present review, this information has so far remained largely unexplored, partly because the low spatial resolution of these surveys is poorly matched to the much finer structure sampled when observing individual stars. But the relevance of hydrogen velocity profiles has already been demonstrated in the specific case of the immediate surroundings of R136 in 30 Doradus (Blades, 1980; Blades and Meaburn, 1980; de Boer, Koornneef and Savage, 1980 ) and in the study of the SMC supergiant Sk143 (Lequeux et al. 1982 ).

THE DUST CONTENT

The mean colour excess in both MCs is rather low. For the SMC, the total mean coloux excess is $E_{B-V}=0.07 \pm 0.04$ of which $0.04 \pm 0.03$ is due to the foreground (Azzopardi and Vigneau, 1976 and references therein). The main absorption lane in the SMC, as defined by de Vaucouleurs (1955), has an "exceptionally high" average $E_{B-V}=0.11$ (in SMC reddening). Similar studies for the LMC, also based on the study of supergiants (Isserstedt, 1975; Brunet, 1975), give $E_{B-V}=0.07 \pm 0.04$ for the foreground with an additional 0.03 to 0.11 within the LMC, dependent on the region.

Very few stars are reddened by more than $E_{B-V}=0.20$ in LMC-dust (Isserstedt, 1975), which is also the upper limit for the effective reddening in the $L M C-O B$ associations measured with OAO-2 (Koornneef, 1984). Reddening in excess of $\mathrm{E}_{\mathrm{B}-\mathrm{V}} \simeq 0.30$ has been claimed by Madore (1976, 1982) for some LMC and SMC Cepheids, but the intrinsic colours of Cepheid variables are rather controversial (e.g. see McNamera and Feltz, 1980; Feast, 1984 and Stift, 1984). Isserstedt (1975) gives a list of LMC supergiants which might have $E_{B-V}>0.30$. Often however, such candidates for high reddening turn out to have been either misclassified, are double or have emission lines (e.g. R 108 in the SMC: Hutchings, 1982; HD37974 in the LMC; Koornneef and Code, 1981).

Three candidates for high reddening in the SMC are BBB275 and 277 (Martin and Thackeray, 1973) and $\operatorname{Sk} 143\left(\mathrm{E}_{\mathrm{B}-\mathrm{V}}=0.27\right.$, Lequeux et al. 1982). Sk 108-69 in the LMC has $E_{B-V} \simeq 0.45$ according to Nandy and Morgan (1978). The dust in front of these last two stars has been shown by these authors to have ultraviolet extinction properties which are different from the average laws in the respective galaxies. 
The statistics of the colour excesses suffer from selection effects as the more heavily reddened stars are naturally fainter and thus easily escape detection. It is thus easy to underestimate the mean reddening as most of the observed stars are only slightly reddened "even in those systems that are optically thick" (Wesselink, 1961). Other proponents of a high ( $A_{v} \geqslant 1 \mathrm{mag}$ ) total dust content (in the SMC) are Hodge (1974) and MacGillivray (1975) who base their findings on background galaxy counts. However, crowding in dense fields might easily lead to an underestimate of the number of background galaxies (e.g. Westerlund, 1974). We will argue later in this review that the actual global gasto-dust ratios in the MC's are indeed substantially in excess of those derived by the use of galaxy counts. But high dust concentrations are likely to exist locally. IRAS observations should be able to pinpoint their location in addition to providing good estimates of the total mass and distribution of the MC dust.

\section{GLOBAL GAS-TO-DUST RATIOS}

As demonstrated in the last section, the evaluation of the mean dust content of the MCs. differs from study to study. In addition, there is some uncertainty with respect to the interpretation of $21 \mathrm{~cm}$ observations. Although clearly penetrating the whole of the external galaxies, the conversion of the measured antenna temperature to column densities can be readily done only under the assumption that the gas is optically thin. Nevertheless, the range in the published estimates in the gas-to-dust ratios is surprisingly large, although all authors who base their values on large scale HI maps and global estimates of the mean colour excesses at least agree that both MCs have more gas relative to dust than our Galaxy (see table 1).

Table 1. Gas to dust ratios relative to the Galactic value

\begin{tabular}{llc}
\hline SMC & van den Bergh (1968) & $>10$ \\
& van Genderen (1969) & $5-12$ \\
& Dachs (1970) & $>100 *$ \\
& Hodge (1974) & 3 \\
& MacGillivray (1975) & 3 \\
& Azzopardi and Vigneau & $15 \quad(9-24)$ \\
& $(1977)$ & 4 \\
LMC & van Genderen (1970) & $20 *$ \\
& Brunet (1975) & $9 *$ \\
\hline
\end{tabular}

* these values have been derived by dividing the total HI column densities by the mean extinction and should therefore be systematically too large. 
GAS TO DUST RATIOS USING HYDROGEN COLUMN DENSITIES FROM LY $\alpha$ ABSORPTION IN INDIVIDUAL SIGHT-LINES

The average galactic gas-to-dust ratio most frequently quoted $\left(\mathrm{N}_{\mathrm{HI}} / \mathrm{E}_{\mathrm{B}-\mathrm{V}}=4.810^{21}\right.$ atoms $\mathrm{cm}^{-2} \mathrm{mag}^{-1} ;$ Bohlin, Savage and Drake, 1978) is derived from the measurement of the interstellar Lyman $\alpha$ atomic hydrogen absorption profile. The Copernicus satellite used by these authors was not sensitive enough for similar measurements of individual stars in external galaxies, but the International Ultraviolet Explorer has now been successfully used for this purpose in both MCs.

A study of Ly $\alpha$ absorption in the IUE spectra of eleven LMC early type supergiants has been published by Koornneef (1982). The total (LMC plus local) colour excesses of these stars range from $\mathrm{E}_{\mathrm{B}-\mathrm{V}}=0.06-0.26$. Under the assumption that the galactic foreground component is the same for each of these stars, a LMC gas to dust ratio of $\mathrm{N}_{\mathrm{HI}} / \mathrm{E}_{\mathrm{B}-\mathrm{V}}=210^{22}$ atoms $\mathrm{cm}^{-2} \mathrm{mag}^{-1}$ is derived.

A similar study for SMC supergiants is being made by Lequeux et al. (1983). Due to the lower mean reddening in the SMC, it is even more difficult to derive accurate gas-to-dust ratios for individual stars than in the LMC. But all of the fifteen stars they are studying have new ground based photometry as well as accurate spectral classifications and Lequeux et al. (1984) conclude that the "normal" SMC gas to dust ratio is approximately 17 times the galactic value.

\section{EXCEPTIONS TO "NORMAL" GAS TO DUST}

Three stars in the SMC sample studied by Lequeux et al. (1984) show gas to dust ratios closer to the average galactic value. They consider them exceptions to the rule and point out that the extinction curves for these stars tend to be more like the average Galactic law. One specific example is Sk143 (Lequeux et al. 1982). Its spectrum shows a significant depression around $2200 \AA$ in contrast with the "average" SMC curve which shows no $2200 \AA$ bump (Rocca-Volmerange et al. 1981, Hutchings 1982, Nandy et al. 1982). Sk143 is the star with the highest colour excess of all objects studied in the SMC. Its "counterpart" in the LMC is Sk108-69 (Nandy and Morgan, 1978) which, with $E_{B-V}=0.45$, suffers more extinction than all other LMC supergiants which have so far been observed with IUE. Its extinction curve is rather more similar to the galactic law than to the "average" LMC curve (Koornneef and Code 1981; Nandy et al. 1981), but regrettably, no gas to dust ratio has so far been derived for this star.

DOES $E_{B-V}$ MEASURE THE AMOUNT OF DUST?

The gas to dust ratios quoted so far are all normalized to unit extinction between $B$ and $V$, and should thus be better described as gas 
to colour excess ratios. One way of explaining the high ratios reported for the MCs is by arguing that the "missing" dust is locked up in grains large enough to have a low visual reddening efficiency. This in fact is the probable explanation of the high $\mathrm{N}_{\mathrm{HI}} / \mathrm{E}_{\mathrm{B}-\mathrm{V}}$ ratio in the Ophiuchus dark cloud (Carrasco, Strom and Strom, 1973). In that region, Whittet and van Breda (1975) also report a high value for the ratio $R$ of total to selective absorption, thus confirming the presence of larger grains. Determinations of $R$ are notoriously difficult and unreliable, but near infrared (JHK) studies in the LMC by Koornneef (1982) and Morgan and Nandy (1982) have not revealed any obvious anomalies in the large particle size distribution function. A similar conclusion has been reached by Clayton and Martin (1984) on the basis of observations of optical linear polarization. For the SMC, Isserstedt (1976, 1980) has derived a surprisingly low value of $R \simeq 2$. This, if confirmed, would indicate a lower than normal number of large particles and this would tend to make the quoted SMC gas-to-dust ratios ever higher if they were expressed as a ratio by mass. Note, however, that Stift (1984) has now argued that the value of $R$ in the SMC substantially exceeds the Galactic value (of $R \simeq 3 \cdot 1$ ).

The very pronounced differences of the ultraviolet part of the extinction curves between the Galaxy, the LMC and the SMC could at least be partly due to changes in the particle size distribution function. Their effect on the normalization of the observed gas to dust ratios deserves further investigation.

\section{SOME LIMITATIONS OF THE PRESENT ULTRAVIOLET DATA}

As the IUE low dispersion Ly $\alpha$ observations do not provide any possibility to discriminate between local and MC gas due to saturation of the profiles, the assumption has to be made that the spatial (i.e. star to star) variations in the foreground gas and dust are very much smaller than their MC counterparts. This might not be quite true as the mean internal reddening in the external galaxies is of the same order of magnitude as the Galactic reddening. If the foreground is not constant it is likely, statistically, that the heavily reddened stars in the sample will have a relatively large foreground component, whereas the stars with the lowest $E_{B-V}$ are likely to suffer little foreground extinction. This would bias the derived gas-to-dust ratios in the direction of the galactic value, i.e. the observed ratios would degrade into a lower limit (a similar argument has been made for MC extinction laws by Koornneef and Code, (1981)\}.

Some concern should also be expressed with respect to the accuracies of the colour excess values as derived from UBV-photometry and spectral types. Most of the MC-supergiants studied so far are intrinsically brighter than their Galactic counterparts and their (ultraviolet) intrinsic colours are likely to be luminosity dependent (e.g. Brunet, 1975; Isserstedt, 1982). Potential errors in this area 
can be minimized by restricting the range in luminosity and spectral type of the sample. This has been carefully considered by Koornneef (1982) and Lequeux et al. (1983) so that their principal results should not be affected. But the study of star-to-star variations of gas-todust ratios (as well as extinction laws) requires more accurate intrinsic colours of early type supergiants as a function of spectral type, luminosity and metallicity. More information is also needed with respect to the relative locations of gas, dust and star along the total (local plus MC) line of sight, both in order to suppress the local effects and to further the study of the multiple components known to exist within the MCs. High resolution optical and ultraviolet interstellar line studies are likely to make important contributions in this area in the near future. It will be 4-5 years, however, before the Australian Synthesis Telescope will start supplying high spatial resolution radio observations.

\section{CONCLUSIONS}

The gas to dust ratios in both Magellanic Clouds are higher than measured in our galaxy by factors of approximately 4 in the LMC and 17 in the SMC. These factors are closely similar to the underabundance of Carbon in the respective Clouds (Dufour, 1983). But the abundances of $S i$ and 0 , which are needed for the formation of silicates, are down with respect to the solar neighbourhood by much smaller factors $(1.8$ in the LMC and 5.8 in the SMC, ; see Lequeux et al. 1979). Empirically, there appears to be a coupling between the gas-to-dust ratios, the metallicity and the characteristics of the ultraviolet interstellar extinction.

Acknowledgements: It is a pleasure to acknowledge very valuable comments by Dr. J. Chris Blades. Ms. Jean Engelke typed and edited the manuscript and Ms. Sarah Stevens-Rayburn provided competent bibliographical assistence.

\section{REFERENCES}

Azzopardi, M., Vigneau, J. 1977, Astron. Astrophys. 56, p.151. Boer, K.S. de, Koornneef, J., Savage, B.D., 1980, Astrophys. J. 236, p. 769.

Blades, J.C. 1980, Mon. Not. Roy. Astr. Soc. 190, p. 33.

Blades, J.C., Meaburn, J. 1980, Mon. Not. Roy• Astr. Soc. 190, p 59P. Bergh, S. van den 1968, J. Roy. Astron. Soc. Canada, 62, p. 145. Bohlin, R.C., Savage, B.D., Drake, J.F., 1978, Astrophys. J. 224,

p. 132 .

Brunet, J.P. 1975, Astron. Astrophys. 43, p. 345.

Carrasco, L., Strom, S.E., Strom, K.M., 1973, Astrophys. J., 182, p. 95. Clayton, G.C., Martin, P.G. 1984, this volume, p. 403.

Dachs, J. 1970, Astron. Astrophys. 9, p. 95.

Dufour, R.J. 1984, this volume, p. 353. 
Feast, M.W. 1984, this volume, p. 157.

FitzGerald, M.P., 1970, Astron. Astrophys. 4, p. 234.

Genderen, A.M. van, 1969, Bull. Astron. Inst. Neth. Suppl. 3, p. 299.

Genderen, A.M. van, 1970, Astron. Astrophys. 7, p. 49.

Hindman, J.V. 1967, Australian J. Phys. 20, p.147.

Hodge, P.W., 1974, Astrophys. J. 192, p. 21.

Hutchings, J.B. 1980, Astrophys. J. 237, p. 285.

Hutchings, J.B. 1982, Astrophys, J. 255, p. 70.

Isserstedt, J. 1975, Astron. Astrophys. 41, p. 175.

Isserstedt, J. 1976, Astron. Astrophys. 47, p. 463.

Isserstedt, J. 1980, Astron. Astrophys. 83, p. 322.

Isserstedt, J. 1982, Astron. Astrophys. 115, p. 97.

Koornneef, J., Code, A.D. 1981, Astrophys. J. 247, p. 860.

Koornneef, J. 1982, Astron. \& Astrophys., 107, p. 247.

Koornneef, J. 1984, this volume, p. 105.

Lequeux, J., Peimbert, M., Rayo, J.F., Serrano, A., Torres-Peimbert, S., 1979, Astron. Astrophys. 80, p. 155.

Lequeux, J., Maurice, E., Prevot-Burnichon, M-L., Prevot, L., RoccaVolmerange, B. 1982, Astron. Astrophys. 113, p. L15.

Lequeux, J., Maurice, E., Prevot, L., Prevot-Burnichon, M-L., RoccaVolmerange, B. 1984, this volume, p. 405.

MacGillivray, H.T. 1975, Mon. Not. Roy. Astr. Soc. 170, p. 241.

Madore, B.F. 1976, Mon. Not. Roy. Astr. Soc. 177, p. 215.

Madore, B.F. 1982, Astrophys. J. 253, p. 575.

Martin, N., Prévot, L., Rebeirot, E., Rousseau, J. 1976, Astron. Astrophys. 51, p.31.

Martin, W.L., Thackeray, A.D. 1973, Mon. Not. Roy. Astr. Soc. 161, p. 5P.

McGee, R.X., Milton, J.A., 1966, Australian J. Phys. 19, p. 343.

McGee, R.X., Newton, L.M., 1981, Proc. Astron. Soc. Aust. 4, p.189.

McGee, R.X., Newton, L.M., 1982, Proc. Astron. Soc. Aust. 4, p.308.

McGee, R.X., Newton, L.M., Morton, D.C. 1983, Mon. Not. Roy. Astr. Soc., submitted.

McNamara, D.H., Feltz, K.A. 1980, Publ. Astron. Soc. Pac. 92, p. 587.

Morgan, D.H., Nandy, K. 1982, Mon. Not. Roy. Astr. Soc. 199, p. 979.

Nandy, K., Morgan, D.H. 1978, Nature 276, p. 478.

Nandy, K., Morgan, D.H., Willis, A.J., Wilson, R., Gondhalekar, P.M., 1981, Monthly Not. Royal Astron. Soc. 196, p. 955.

Nandy, K., McLachlan, A., Thompson, G.I., Morgan, D.H., Willis, A.J., Wilson, R., Gondhalekar, P.M., Houziaux, L. 1982, Mon. Not. Roy. Astr. Soc. 201, p. 1p.

Rocca-Volmerange, B., Prévot, L., Ferlet, R., Lequeux, J., PrévotBurnichon, M.L. 1981, Astron. \& Astrophys. 99, p. L5.

Stift, M.J. 1984, this volume, p. 229.

vaucouleurs, G. de 1955, Astron. J. 60, p. 219.

Wesselink, A.J. 1961, Mon. Not. Roy. Astr. Soc. 122, p. 509.

Westerlund, B.E. 1974, Proc. 1st European Astr. Meeting. Vol. III, p. 39 .

Whittet, D.C.B., van Breda, I.G. 1975, Astrophys. Space Sci. 38, L3. Whittet, D.C.B., van Breda, I.G., 1978, Astron. Astrophys. 66, p. 57. 